\title{
Analysis of reactant gas transport in a PEM fuel cell with partially blocked fuel flow channels
}

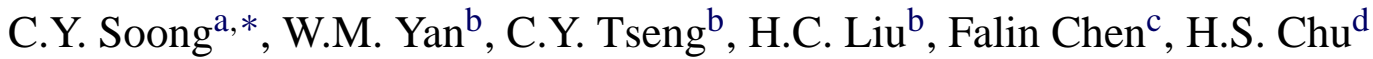 \\ ${ }^{a}$ Department of Aerospace and System Engineering, Feng Chia University, 100 Wenhua Road, Seatwen, Taichung 40724, Taiwan, ROC \\ ${ }^{\mathrm{b}}$ Department of Mechatronic Engineering, Huafan University, Shih-Ting, Taipei 223, Taiwan, ROC \\ ${ }^{\mathrm{c}}$ Institute of Applied Mechanics, National Taiwan University, Tapei 107, Taiwan, ROC \\ ${ }^{\mathrm{d}}$ Energy and Resources Lab, Industrial Technology Research Institute, Chutung, Hsin-Chu 310, Taiwan, ROC
}

Received 30 May 2004; received in revised form 4 November 2004; accepted 9 November 2004

Available online 2 February 2005

\begin{abstract}
In the present study, we propose a novel configuration of partially blocked fuel channel with baffle plates transversely inserted in the channel. The effects of the blockage with various gap ratios and numbers of the baffle plates, the fuel flow Reynolds number and the porosity of the diffusion layer on the reactant gas transport, and the pressure drop across the channel length are explored. A two-dimensional model of one-side porous-walled channel with the presence of baffle plates is considered. An approximate analysis is performed first to examine the qualitative natures of the flow in the gap region and then numerical simulations for the parametric study of the reactant gas transport in a half-cell model are carried out. The velocity field, the oxygen mass flux/flow rate reaching the catalyst layer, the concentration of the water vapor produced, the fraction of the fuel gas entering the diffusion layer, and the pressure drop at various conditions are analyzed. The results reveal that reducing the gap size and/or increasing the baffle number to enhance the reactant gas transport results in a penalty of high pressure-loss. Among the parameters considered in the present work, the gap ratio has the most remarkable impact on the variation of the pressure drop. Very high pressure-loss can be generated due to high flow resistance at a low gap ratio combined with a low gas diffusion layer (GDL) porosity. With the consideration of both high performance and reasonable pressure drop, the present results disclose that designs with the baffle gap ratio no smaller than 0.1 , number of baffle plates $N=3-5$, and the GDL porosity around 0.7 seem quite appropriate.
\end{abstract} (C) 2004 Elsevier B.V. All rights reserved.

Keywords: Proton exchange membrane fuel cell; Partially blocked fuel channel; Reactant gas transport; Pressure drop; Diffusion in porous layer

\section{Introduction}

Design of the flow channel in bipolar plates is one of the key factors in the system performance issue of proton exchange membrane (PEM) fuel cell systems. The section geometry and the pattern of the fuel flow channel influence the reactant gas transport, water management, and the efficiency of the utilization of the fuel. If the behaviors of the exhaust gas and waste water are out of control, the supply of oxygen will be prohibited, which in turn may cause overheat and dryout of the fuel cell system. This unfavorable situation will

\footnotetext{
* Corresponding author. Fax: +886 424516246.

E-mail address: cysoong@fcu.edu.tw (C.Y. Soong).
}

lead to the increase in the inter-resistance and deterioration in the system performance. On the contrary, an appropriate design of fuel flow channel is beneficial to the reactant gas transport as well as the water management.

In the past years, therefore, lots of efforts have been endeavored to novel flow channels in PEM fuel cell systems such as the arrangements of serpentine channel, multiple channels in parallel type, interdigited channels, etc. Since the fuel channel has gas diffusion layer (GDL) as a side-wall, the GDL morphology may affect the reactant gas transport from the channel to the catalyst surface and is usually included in dealing with the gas transport. The geometric parameters have been analyzed in order to optimize the fuel cell system performance. The effects of the rib spacing of the fuel chan- 


\begin{tabular}{|c|c|}
\hline \multicolumn{2}{|c|}{ Nomenclature } \\
\hline$a$ & coefficient \\
\hline$A, B, C$ & constants \\
\hline$C_{\mathrm{F}}$ & quadratic drag factor \\
\hline$D_{1}$ & mass diffusivity of oxygen $\left(\mathrm{m}^{2} \mathrm{~s}^{-1}\right)$ \\
\hline$D_{2}$ & mass diffusivity of vapor $\left(\mathrm{m}^{2} \mathrm{~s}^{-1}\right)$ \\
\hline$E_{\mathrm{a}}$ & activation energy $\left(\mathrm{J} \mathrm{mol}^{-1}\right)$ \\
\hline$h_{1}$ & flow channel height (m) \\
\hline$h_{2}$ & gap between baffle and diffusion layer ( $\mathrm{m}$ ) \\
\hline$h_{3}$ & thickness of diffusion layer (m) \\
\hline$k$ & permeability $\left(\mathrm{m}^{2}\right)$ \\
\hline$L$ & flow channel length (m) \\
\hline$L_{2}$ & baffle plate thickness (m) \\
\hline$\dot{m}$ & mass flow rate $\left(\mathrm{kg} \mathrm{s}^{-1}\right)$ \\
\hline$\dot{m}_{1}^{\prime \prime}$ & molar mass flux of oxygen $\left(\mathrm{kg} \mathrm{m}^{-2} \mathrm{~s}^{-1}\right)$ \\
\hline$M$ & molar mass $\left(\mathrm{g} \mathrm{mol}^{-1}\right)$ \\
\hline$N$ & number of baffle plates \\
\hline$P$ & pressure $\left(\mathrm{N} \mathrm{m}^{-2}\right)$ \\
\hline$R$ & gas constant $\left(\mathrm{J} \mathrm{mol}^{-1} \mathrm{~K}^{-1}\right)$ \\
\hline $\operatorname{Re}$ & Reynolds number, $U_{0} h_{1} / v$ \\
\hline$t$ & time (s) \\
\hline$T$ & temperature $(\mathrm{K})$ \\
\hline$U$ & velocity in $x$-direction $\left(\mathrm{m} \mathrm{s}^{-1}\right)$ \\
\hline$U_{0}$ & average velocity at inlet $\left(\mathrm{m} \mathrm{s}^{-1}\right)$ \\
\hline $\bar{U}$ & $\begin{array}{l}\text { average velocity at center of the baffle gap, } \\
U_{0} h_{1} /\left(h_{2}+h_{3}\right)\left(\mathrm{m} \mathrm{s}^{-1}\right)\end{array}$ \\
\hline$V$ & velocity in $y$-direction $\left(\mathrm{m} \mathrm{s}^{-1}\right)$ \\
\hline & molar mass fraction of oxygen \\
\hline & molar mass fraction of vapor \\
\hline$X, Y$ & cartesian coordinates (m) \\
\hline \multicolumn{2}{|c|}{ Greek letters } \\
\hline$\alpha$ & Beavers-Joseph constant \\
\hline$\varepsilon$ & porosity \\
\hline$\lambda$ & gap ratio, $h_{2} / h_{1}$ \\
\hline$v$ & kinematic viscosity $\left(\mathrm{m}^{2} \mathrm{~s}^{-1}\right)$ \\
\hline$\rho$ & density $\left(\mathrm{kg} \mathrm{m}^{-3}\right)$ \\
\hline \multicolumn{2}{|c|}{ Subscripts } \\
\hline 0 & inlet \\
\hline 1 & oxygen \\
\hline & vapor \\
\hline $\mathrm{b}$ & boundary \\
\hline & channel \\
\hline $\mathrm{d}$ & porous diffusion layer \\
\hline & effective \\
\hline & $i$ th species \\
\hline
\end{tabular}

nels and the GDL thickness on the cell performance were studied by West and Fuller [1]. By conducting experiments with a model of hydrophobilicity, Jordan et al. [2,3] studied and explained the effects of the GDL morphology on the cell performance. Gurau et al. [4] proposed a mathematical model to obtain an analytic solution of the mass transport of reactant gas in a half-cell, in which the effects of the porosity and the tortuosity of the gas diffusion layer and the catalyst layer were explored.

Recently, numerical computations for details of the flow distribution and fuel gas diffusion have been performed. In their simulation, Hontanon et al. [5] focused their attention on the flow distributor to search for optimization of the fuel flow characteristics. They employed the Navier-Stokes equations and the Michaelis-Menten type two-step kinetics model to study the performance of the grooved plate and porous flow distributors at anode. The predictions revealed that the fuel consumption increases with decreasing permeability of the flow distributor. With the consideration of the reactant gas utilization under the same pressure drop, they also found that the porous materials are more advantageous than the grooved plates. Dutta et al. [6] performed three-dimensional computations for fuel transport in fuel cells of serpentine channels with the electrochemistry modeled by the mass source/sink terms. Kee et al. [7] proposed a generalized computational model for the mass and momentum transport in channel networks of typical planar fuel cell layers/stacks. Numerical simulation was performed for a PEM fuel cell with a metal foam in the flow field of the bipolar plates [8] and the results demonstrated that the use of metal foam renders the local current density distribution more uniform. Yan et al. [9] conducted a numerical study of the effects of fuel channel width and GDL porosity on the cross-cell transport of reactant gas and the performance of a PEM fuel cell. Most recently, GDL characteristics with various thickness and porosity fabricated by using different manufacturing methods were studied by numerical simulation and experimental measurements [10].

From the above brief survey, it is found that the fuel flow channel design is very significant to the performance of the PEM fuel cells and various configurations have been studied previously. The interdigited channel with fuel flow fully blocked can result in a satisfactory performance in reactant gas transport. In the previous investigations, however, the penalty of high pressure-loss was not discussed in detail. The objective of the present work is to (1) propose a novel configuration of partially blocked fuel channel; (2) explore the influences of the blockage with various gap ratios and numbers of the baffle plates on the reactant gas transport; (3) evaluate the pressure drop in the presence of various blockage effects; and (4) examine the influences of fuel flow Reynolds number and porosity of the gas diffusion layer. A two-dimensional model of one-side porous-walled channel with baffle plate(s) in it is considered. The hydrodynamic nature is the main theme of the study under the assumption of fast reaction at the surface of the catalyst layer. To qualitatively examine the hydrodynamic natures and used as a limiting case for numerical verification, an approximate analysis of the flow in gap region beneath a baffle plate is performed first. Then the numerical simulation is carried out for parametric study of the mass/momentum transport in the whole model by utiliz- 
ing the commercial software CFDRC-ACE+. The velocity field, the oxygen mass flux/flow rate reaching at the catalyst layer, the concentration of water vapor produced, the mass flow fraction of the fuel gas entering the diffusion layer, and the pressure drop at various conditions are analyzed.

\section{Half-cell model for PEM fuel cells}

\subsection{Governing equations}

Fig. 1 shows the present two-dimensional half-cell model of PEMFC systems for the fuel gas mass and momentum transport in the flow channel and the porous GDL. The fluid in the channel is air at inlet pressure $P_{0}$ and velocity $U_{0}$. The operating temperature is set to be $T_{0}=333 \mathrm{~K}$ in the simulation. The channel length is $L=L_{1}+L_{2}+L_{3}$ with $L_{2}$ standing for the thickness of the baffle plate and $L_{1}$ and $L_{3}$ for the upstream and downstream channel lengths. The parameters $h_{1}, h_{2}$ and $h_{3}$ denote flow channel height, gap size and GDL thickness, respectively. To characterize the blockage effect, a dimensionless parameter named gap ratio, $\lambda \equiv h_{2} / h_{1}$, is defined. The values of $\lambda=0$ and 1 indicate the fully blocked and block-free cases, respectively, and the values in between measure various levels of blockage. The flow is assumed steady, laminar and incompressible, the porous layer is of isotropic and uniform material with the porosity $\varepsilon$ and the permeability $k$. With the assumption of fast reaction, i.e., the chemical reaction time scale smaller than the transport time scale, the hydrodynamic nature plays a dominant role in the process and the chemical reaction is expressed simply as a boundary condition at the surface of the catalyst layer. Since the catalyst layer is extremely thin and, therefore, is simply regarded as a boundary condition, for which the reactant gas (air in this case) is exhausted for perfect reaction. Under the above assumptions, the governing equations of mass, momentum and species balance can be depicted as follows $[11,12]$.

(A) In the flow channel:

$$
\begin{aligned}
& \frac{\partial U}{\partial X}+\frac{\partial V}{\partial Y}=0 \\
& U \frac{\partial U}{\partial X}+V \frac{\partial U}{\partial Y}=-\frac{1}{\rho} \frac{\partial P}{\partial X}+v\left(\frac{\partial^{2} U}{\partial X^{2}}+\frac{\partial^{2} U}{\partial Y^{2}}\right)
\end{aligned}
$$

$$
\begin{aligned}
& U \frac{\partial V}{\partial X}+V \frac{\partial V}{\partial Y}=-\frac{1}{\rho} \frac{\partial P}{\partial Y}+v\left(\frac{\partial^{2} V}{\partial X^{2}}+\frac{\partial^{2} V}{\partial Y^{2}}\right) \\
& U \frac{\partial W_{i}}{\partial X}+V \frac{\partial W_{i}}{\partial Y}=D_{i}\left(\frac{\partial^{2} W_{i}}{\partial X^{2}}+\frac{\partial^{2} W_{i}}{\partial Y^{2}}\right)
\end{aligned}
$$

where $W_{i}$ and $D_{i}$ denote mass fraction and diffusivity, respectively, the subscript $i=1$ stands for oxygen and $i=2$ for water vapor.

(B) In the gas diffusion layer:

$$
\begin{aligned}
\frac{\partial U}{\partial X}+\frac{\partial V}{\partial Y}=0 & \\
\varepsilon\left(U \frac{\partial U}{\partial X}+V \frac{\partial U}{\partial Y}\right)= & -\frac{\varepsilon}{\rho} \frac{\partial P}{\partial X}+v \varepsilon\left(\frac{\partial^{2} U}{\partial X^{2}}+\frac{\partial^{2} U}{\partial Y^{2}}\right) \\
& -\frac{v \varepsilon^{2}}{k} U-\frac{\varepsilon^{3} C_{\mathrm{F}} \rho U}{\sqrt{k}} \sqrt{U^{2}+V^{2}}
\end{aligned}
$$

$$
\begin{aligned}
\varepsilon\left(U \frac{\partial V}{\partial X}+V \frac{\partial V}{\partial Y}\right)= & -\frac{\varepsilon}{\rho} \frac{\partial P}{\partial Y}+v \varepsilon\left(\frac{\partial^{2} V}{\partial X^{2}}+\frac{\partial^{2} V}{\partial Y^{2}}\right) \\
& -\frac{v \varepsilon^{2}}{k} V-\frac{\varepsilon^{3} C_{F} \rho V}{\sqrt{k}} \sqrt{U^{2}+V^{2}}
\end{aligned}
$$

$\varepsilon\left(U \frac{\partial W_{i}}{\partial X}+V \frac{\partial W_{i}}{\partial Y}\right)=D_{i, \text { eff }}\left(\frac{\partial^{2} W_{i}}{\partial X^{2}}+\frac{\partial^{2} W_{i}}{\partial Y^{2}}\right)$

In Eqs. (6) and (7), $C_{\mathrm{F}}$ is the quadratic drag factor, and Blake-Kozeny correlation [13] is adopted for the relationship between the porosity $\varepsilon$ and the permeability $k$, viz.,

$k=\left(\frac{D_{\mathrm{d}}^{2}}{150}\right)\left[\frac{\varepsilon^{3}}{(1-\varepsilon)^{2}}\right]$

$D_{\mathrm{d}} \equiv \frac{6 V_{\mathrm{d}}^{\prime}}{S_{\mathrm{d}}^{\prime}}$

In the above equations, the quantities with the subscript $d$ denote the quantities in the porous diffusion layer. The geometry parameter $V_{\mathrm{d}}^{\prime} / S_{\mathrm{d}}^{\prime}$ is the volume-to-surface ratio of the GDL. The last two terms in Eqs. (6) and (7) are the drag forces stemmed from the presence of the porous wall and may result in an increase in pressure drop. The parameter $D_{i, \text { eff }}$ is

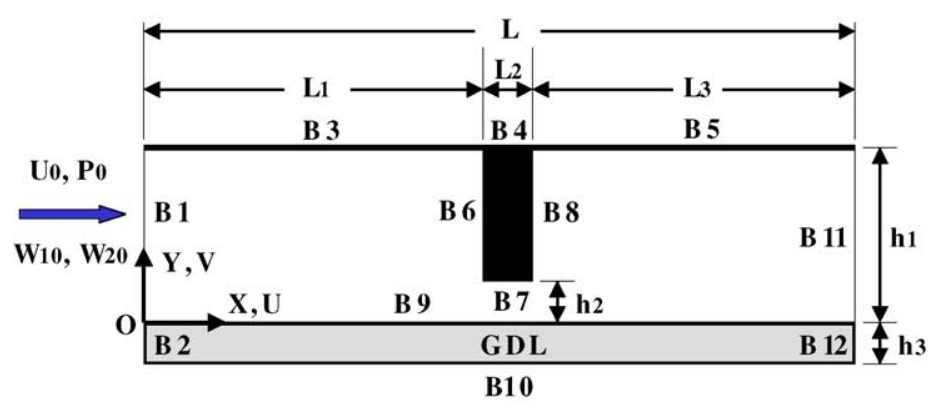

Fig. 1. PEMFC half-cell model of partially blocked flow channel. 
the effective diffusivity of the $i$ th gas component in GDL and the Bruggemann correlation [14],

$D_{i, \text { eff }}=D_{i} \cdot \varepsilon^{1.5}$

is adopted.

\subsection{Boundary conditions}

As that shown in Fig. 1, the conditions at the boundaries designated by B1-B12 are listed as follows:

At B1:

$U=U_{0}, \quad V=0, \quad P=P_{0}$,

$W_{1}=W_{1,0}, \quad W_{2}=W_{2,0}$

At B2, B6, B8, B12:

$U=V=\frac{\partial W_{i}}{\partial X}=0$

At B3, B5, B7:

$U=V=\frac{\partial W_{i}}{\partial Y}=0$

At B4:

$U=V=W_{i}=0$,

At B9:

$\left.\frac{\partial U}{\partial Y}\right|_{Y=h_{3}^{+}}=\left.\varepsilon \frac{\partial U}{\partial Y}\right|_{Y=h_{3}^{-}},\left.\quad \frac{\partial V}{\partial Y}\right|_{Y=h_{3}^{+}}=\left.\varepsilon \frac{\partial V}{\partial Y}\right|_{Y=h_{3}^{-}}$,

$\left.\frac{\partial W_{i}}{\partial Y}\right|_{Y=h_{3}^{+}}=\left.\varepsilon \frac{\partial W_{i}}{\partial Y}\right|_{Y=h_{3}^{-}}$

At B10:

$U=V=W_{1}=0$,

$\frac{\partial W_{2}}{\partial Y}=\rho B^{\prime} \mathrm{e}^{-\left(E_{\mathrm{a}} / R T\right)} \sqrt{\frac{R T}{2 \pi M_{1}}} \times \frac{D_{1, \mathrm{eff}}}{D_{2, \mathrm{eff}}} \times \frac{\partial W_{1}}{\partial Y}$

At B11:

$\frac{\partial U}{\partial X}=\frac{\partial V}{\partial X}=\frac{\partial W_{i}}{\partial X}=0$

Since the fast and complete reaction of oxygen is assumed at the interface of the GDL and the catalyst layer, the relation of the oxygen and water vapor can be described by Eq. (12f), where $B^{\prime}$ is a constant, $E_{\mathrm{a}}$ is the activation energy, $R$ is the gas constant, and $M_{1}$ is the molar weight of oxygen.

\subsection{Numerical procedure}

The system of the governing equations described above is solved utilizing the commercial code CFD-ACE+ based on the SIMPLE Algorithm [15] for convection-diffusion problems. Before mass data generation, a preliminary numerical

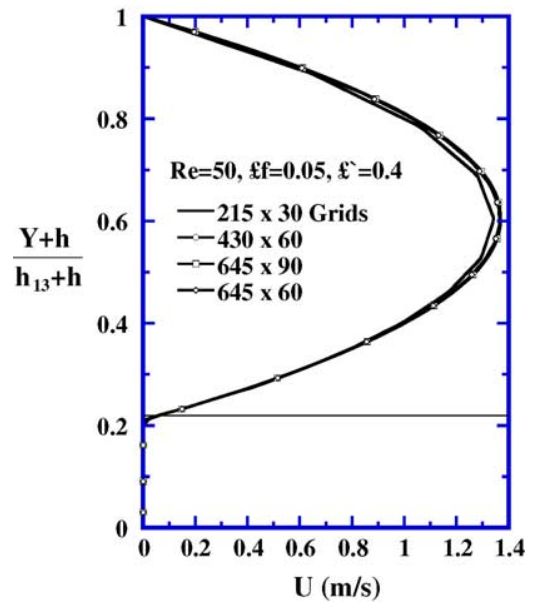

Fig. 2. Grid-dependence test by examining the axial velocity distributions at $X / L=0.25$.

test was performed to validate the computations and examine the grid-dependence of the results. By employing a baseline case of Reynolds number $R e=50$, one baffle plate $N=1$, gap ratio $\lambda=0.05$, and porosity $\varepsilon=0.4$, computations on the four grid systems with number of grid points (length $\times$ width) $215 \times 30,430 \times 60,645 \times 60$, and $645 \times 90$ were performed. The axial velocity profiles at $X / L=0.25$ are plotted and compared in Fig. 2. It can be observed that the grid of $430 \times 60$ points is appropriate with the considerations of accuracy and time-saving.

\section{Approximate solution of flow in a narrow gap region with one-side porous wall}

Fig. 1 shows the two-dimensional flow channel model with the narrow gap region $-h_{3} \leq Y \leq h_{2}$ beneath the baffle plate. To facilitate an approximate analysis, assume that Darcy law [16] can be used to describe the fluid flow in the porous GDL. The governing equation of the flow in this composite channel configuration can be written as

(A) In the gap region of the flow channel $0 \leq Y \leq h_{2}$ :

$\nabla \cdot \boldsymbol{V}=0$

$\frac{\partial \boldsymbol{V}}{\partial t}+\boldsymbol{V} \cdot \nabla \boldsymbol{V}=-\frac{1}{\rho} \nabla P+v \nabla^{2} \boldsymbol{V}$.

(B) In the diffusion layer $-h_{3} \leq Y \leq h_{2}$ :

$\nabla \cdot V_{d}=0$

$\frac{1}{\varepsilon} \frac{\partial \boldsymbol{V}_{\mathrm{d}}}{\partial t}=-\frac{1}{\rho} \nabla P_{d}-\frac{\nu}{k} \boldsymbol{V}_{\mathrm{d}}$,

The related boundary conditions are:

At $Y=h_{2}: V=(U, V)=0$. 


$$
\begin{gathered}
\text { At } Y=0: \frac{\partial U}{\partial Y}=\frac{\varepsilon}{\sqrt{k}}\left(U-U_{\mathrm{d}}\right) ; \quad V=V_{\mathrm{d}} \\
P_{\mathrm{d}}-P+2 \mu \frac{\partial V}{\partial Y}=0 .
\end{gathered}
$$

At $Y=-h_{3}: V_{\mathrm{d}}=0$.

For very narrow gap, i.e., $\lambda \ll 1$, the flow can be assumed fully-developed in the narrow gap. The above momentum equation can be reduced to the form of

$-v \frac{\mathrm{d}^{2} U}{\mathrm{~d} Y^{2}}=-\frac{1}{\rho} \frac{\partial P}{\partial X}=A$.

Integrating the last equation twice gives the following solution:

$U=-\frac{A Y^{2}}{2 v}+B Y+C$.

From Eq. (16) with $-(1 / \rho) \partial P / \partial X=A$, the fluid velocity in GDL becomes

$U_{\mathrm{d}}=\frac{k}{v} A$.

With the above boundary conditions, the constants $B$ and $C$ in Eq. (21) can be determined,

$B=\frac{A h_{2}}{v} L, \quad C=\frac{A h_{2}^{2}}{v} M$,

in which

$$
\begin{aligned}
& L=\frac{\alpha \hat{d}}{2(\delta+\alpha \hat{d})}+\frac{\alpha^{2} \delta}{\delta+\alpha \hat{d}}-\frac{\alpha \delta}{\hat{d}} ; \\
& M=\frac{\delta}{2(\delta+\alpha \hat{d})}+\frac{\alpha \delta^{2}}{\hat{d}(\delta+\alpha \hat{d})} ; \quad \delta=\frac{\sqrt{k}}{h_{3}} ; \quad \hat{d}=\frac{h_{2}}{h_{3}} .
\end{aligned}
$$

The fluid velocity in the channel can be expressed as

$$
U(Y)=\frac{h_{2} A}{v}\left(-\frac{Y^{2}}{2}+L Y+h_{2} M\right) .
$$

The fraction of the mass flow rate through the diffusion layer, $\dot{m}_{d}$, with respect to the total mass flow rate, $\dot{m}_{o}$, is also an important parameter in design, and can be evaluated by combining Eqs. (21) and (24), viz.

$$
\frac{\dot{m}_{\mathrm{d}}}{\dot{m}_{\mathrm{o}}}=\frac{\dot{m}_{\mathrm{d}}}{\dot{m}_{\mathrm{d}}+\dot{m}_{\mathrm{c}}}=\frac{k h_{3}}{k h_{3}+h_{2}^{3}[-(1 / 6)+(L / 2)+M]}
$$

The parameter $\dot{m}_{\mathrm{c}}$ stands for the mass flow rate through the flow channel. With the relation of $h_{2}=\lambda h_{1}$, Eq. (26) can be written as

$$
\frac{\dot{m}_{\mathrm{d}}}{\dot{m}_{\mathrm{o}}}=\frac{\dot{m}_{\mathrm{d}}}{\dot{m}_{\mathrm{d}}+\dot{m}_{\mathrm{c}}}=\frac{k h_{3}}{k h_{3}+\lambda^{3} h_{1}^{3}[-(1 / 6)+(L / 2)+M]}
$$

\section{Results and discussion}

\subsection{Effects of GDL porosity on flow and mass transfer}

In the following discussion, a baseline case of $R e=50$, $\lambda=0.05$, and $\varepsilon=0.4$ is defined in the parametric analysis. To examine effects of the porosity, the values of $\varepsilon=0.1,0.4,0.7$, and 0.9 are considered. For the typical cases of $R e=50, N=1$, and $\lambda=0.05$ in Fig. 3 , the close-up views of the velocity vector fields in the baffle-gap-GDL region of the present composite flow passage clearly demonstrate the increase in mass flow rate through GDL of high porosity. At a low porosity, the gas flow moves downstream mainly through the gap between the baffle and the GDL at a high speed, thereby a recirculation appears behind the baffle. This recirculation bubble may be reduced in size by increasing the GDL porosity. For example, with the flows in the cases of $\varepsilon=0.4$ and 0.7 compared with that of $\varepsilon=0.1$, the lower velocity at the exit of the narrow gap and the pushing effect of the flow out of the GDL both tend to deflect the flow behind the block upward and reduce the size of the recirculation bubble. It is most remarkable in the case of $\varepsilon=0.7$. However, as the porosity approaches a value close to unity, e.g., $\varepsilon=0.9$, the flow velocity slip at the interface, $y=0$, can be alleviated. The deflecting or pushing effect stemmed from the gas flow out of the GDL becomes weak and the size of the recirculation bubble can be enlarged to a certain degree. These flow characteristics may be related to the pressure drop of the channel flow.

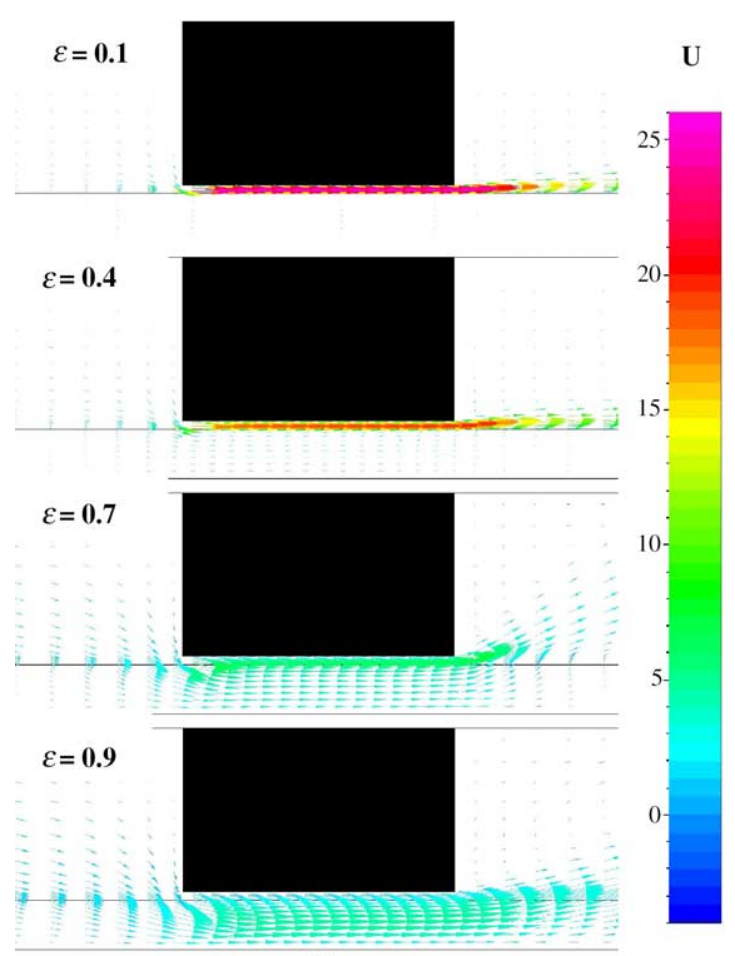

(a)

Fig. 3. Close-up views of the velocity vector field near the gap of the baffle plate in the baffle-gap-GDL region of the present composite flow passage of one baffle at $R e=50$ and $\lambda=0.05$. 

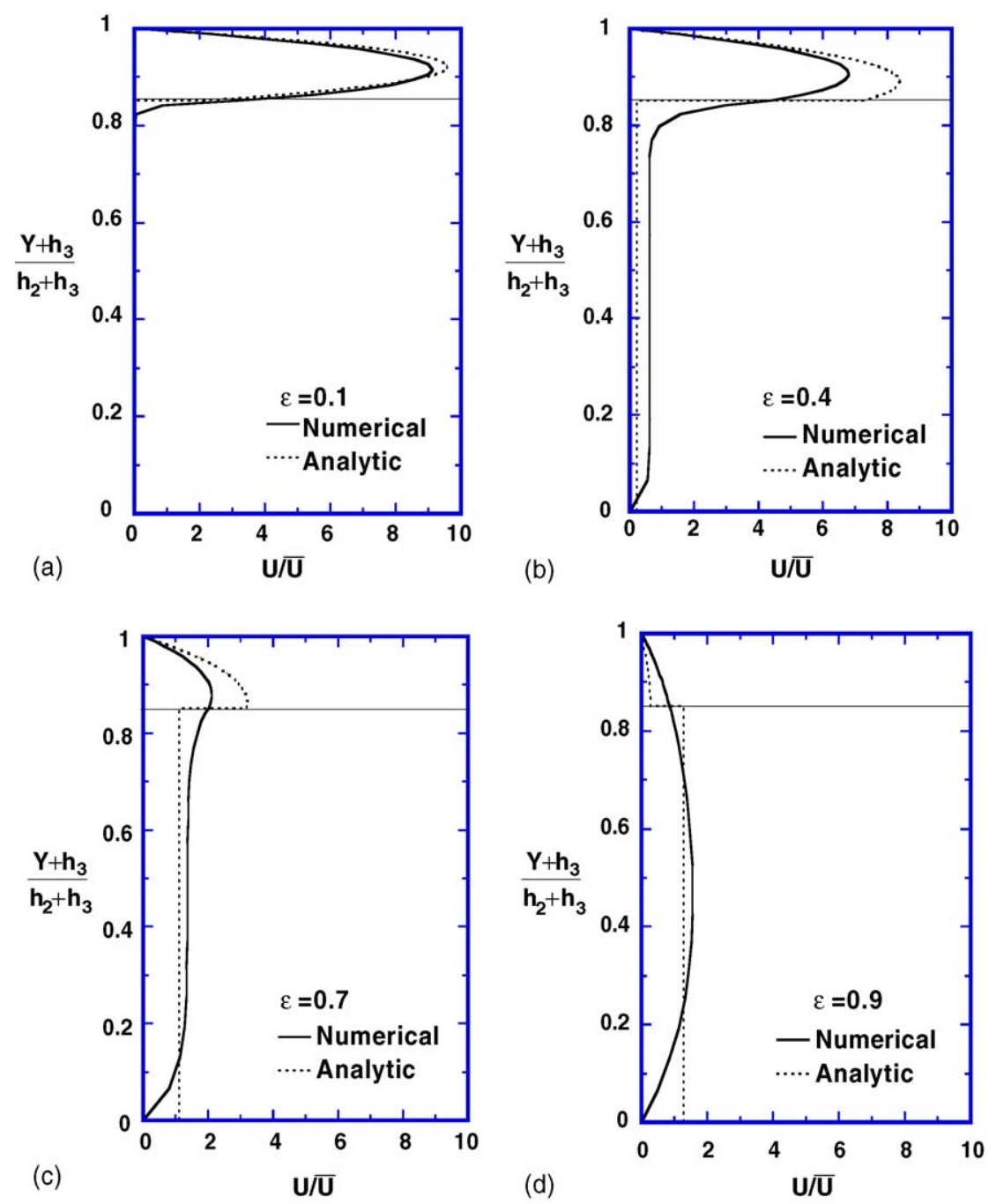

Fig. 4. Comparisons of numerical and analytical results of axial velocity distributions in the gap region and porous GDL layer at $R e=50$ and $\lambda=0.05$. (a) $\varepsilon=0.1$; (b) $\varepsilon=0.4$; (c) $\varepsilon=0.7$; (d) $\varepsilon=0.9$.

At $R e=50$ and $\lambda=0.05$, Fig. 4 presents comparisons of numerical and analytical results of axial velocity profiles at the midway of the gap region, i.e., $X=L_{1}+L_{2} / 2$. The thin line of $Y \approx 0.85$ indicates the interface of the flow channel and the GDL. For the small values of the porosity, e.g., $\varepsilon=0.1$, most of the fuel gas passes axially through the gap region. However, with increasing porosity, more gas enters the porous diffusion layer for the lower flow resistance. Therefore, the fluid velocity and the corresponding mass flow rate in the GDL increase. Although the quantitative deviations exist, both the approximate analysis and the computational results show the same trend and the agreement at low porosity is better. The Darcy law gives a uniform flow solution the in porous layer, which is a good approximation only for the case of low porosity. The analytical approximation is restricted by the validation of the Darcy law with noticeable discrepancy at higher porosity.

Fig. 5 reveals the effects of GDL porosity on the concentration contours of the oxygen and water vapor. The model under consideration is the half-cell on the cathode side, where the oxygen and the water vapor concentrations, as shown in Fig. 5(a) and (b), are the major concerns in the mass transport aspect. Fig. 5(a) shows the oxygen contours corresponding to the cases in Fig. 3. In the case of the low porosity $\varepsilon=0.1$, most of the oxygen appears in the flow channel, only very little oxygen can transport through the GDL and reach the catalyst layer. This leads to a low reaction rate and therefore a low system performance. As the porosity increases, e.g. $\varepsilon=0.4,0.7$ and 0.9 , more oxygen enters the porous layer and the oxygen concentration decreases obviously along the channel length. The reaction at the catalyst surface is enhanced with more fuel supplied. In the region beneath the baffle plate, a large amount of oxygen is forced to turn into the porous GDL and strong reaction and higher consumption of oxygen occur in this region.

Water is the product of the reaction at the surface of the catalyst layer, therefore, the maximum water vapor concentration can be observed at the interface of the GDL and the 

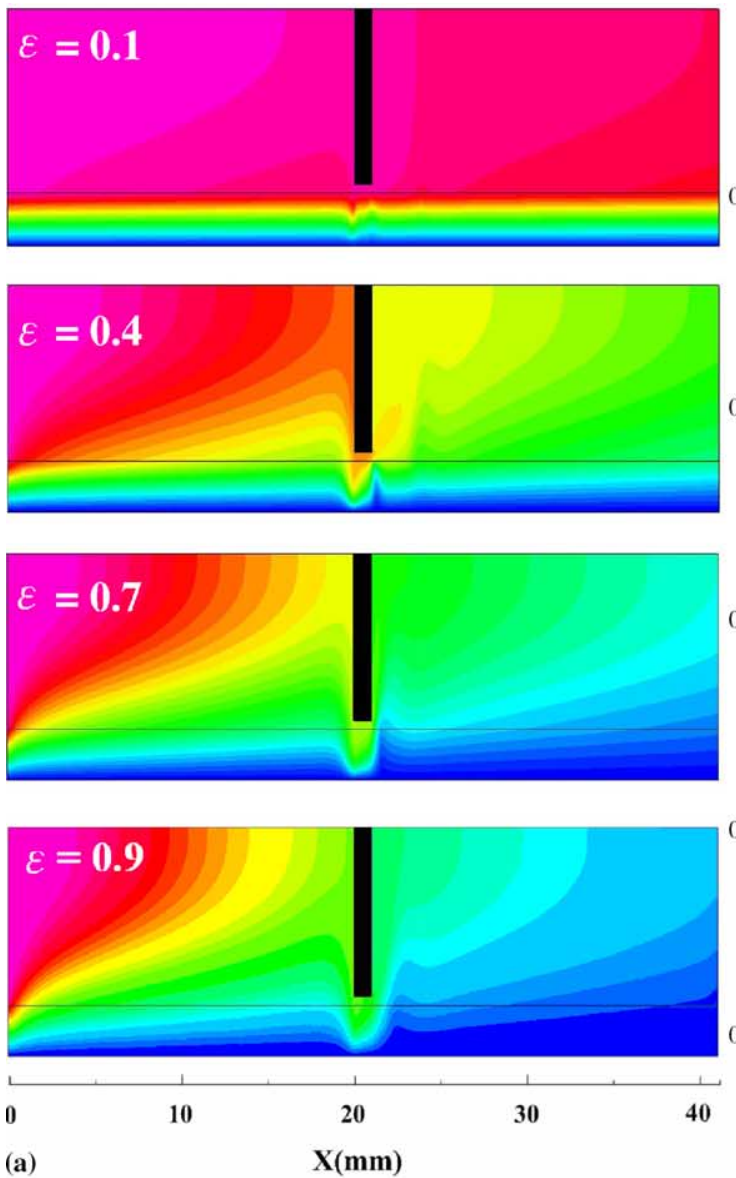

(a)

$\mathbf{X}(\mathbf{m m})$
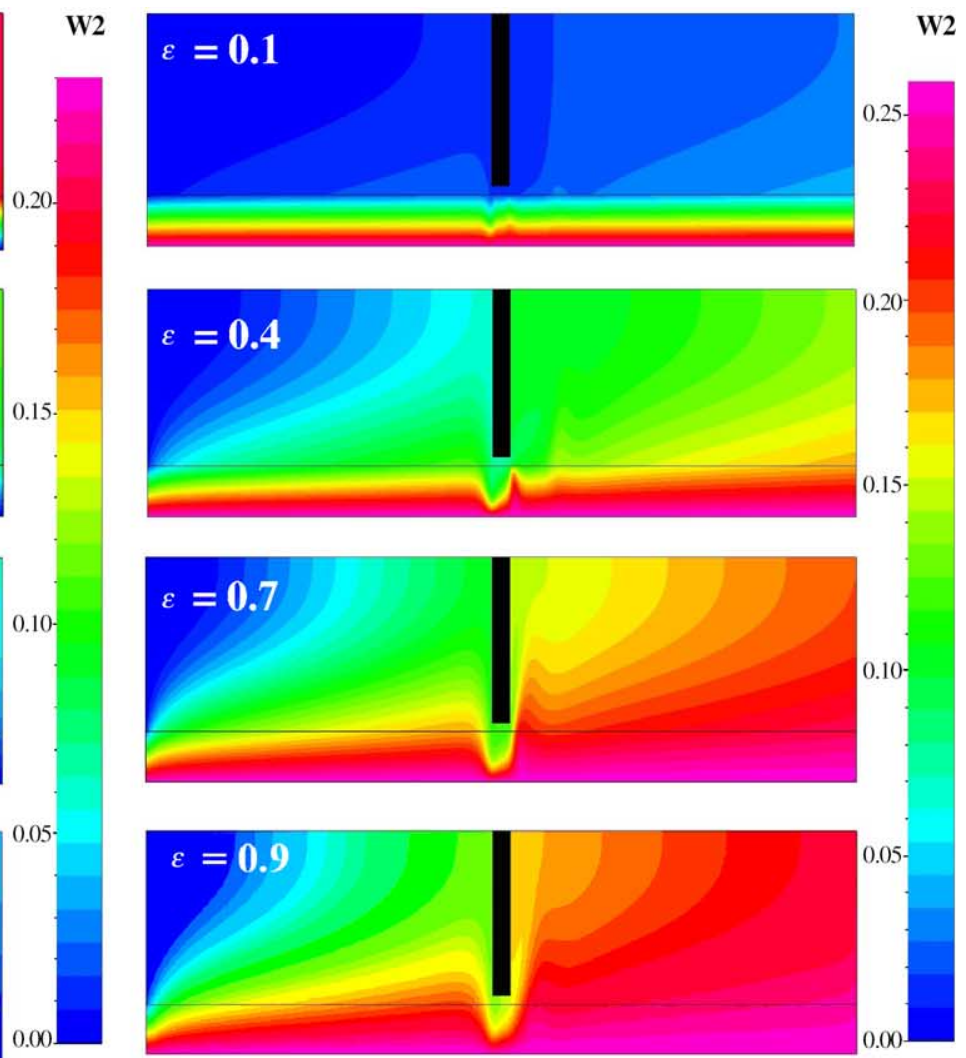

$\mathbf{0}$

(b)
20

$\mathrm{X}(\mathrm{mm})$

Fig. 5. Effects of porosity on the local oxygen and water vapor concentration in the baffle-gap-GDL region of the present composite flow passage of one-baffle at $R e=50$ and $\lambda=0.05$. (a) Oxygen; (b) water vapor.

catalyst layer where the oxygen is consumed. For low GDL porosity, the reactant gas transport through the GDL is difficult and the reaction is weak. However, the situation can be improved by increasing the porosity of the GDL. Fig. 5(b) demonstrates an increasing water vapor transport from the catalyst layer to the flow channel with porosity. Therefore, high porosity is beneficial to remove the excess water. In Fig. 5(a) and (b), it is also observed that the concentrations of the oxygen (reactant) and the water vapor (product) are of an approximately complementary relation. The higher water concentration appears at the location where there is less oxygen, and vice versa.

At the catalyst surface, the effects of GDL porosity on the axial distribution of the oxygen mass flux are displayed in Fig. 6. In general the oxygen decreases along the axial length but with an abrupt jump in the region beneath the baffle plate, where the strong forced convection enhances the transport of the oxygen as mentioned previously. The peak of the mass flux jump is raised up in the cases of high porosity, e.g., $\varepsilon=0.7$ and 0.9 . Behind the baffle plate, however, the oxygen fluxes for high porosity become less due to the stronger reaction with fuel depletion occurs upstream.

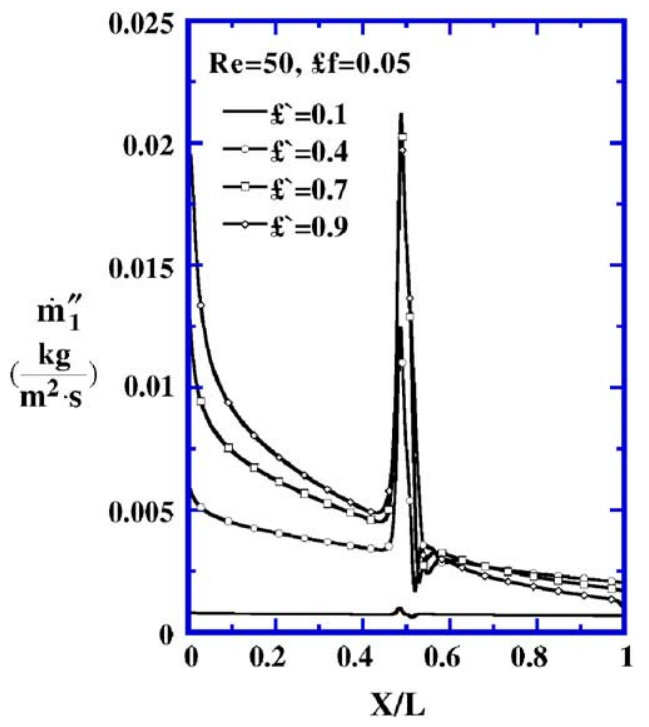

Fig. 6. Effects of porosity on the axial distribution of oxygen mass fluxes at the surface of the catalyst layer. 

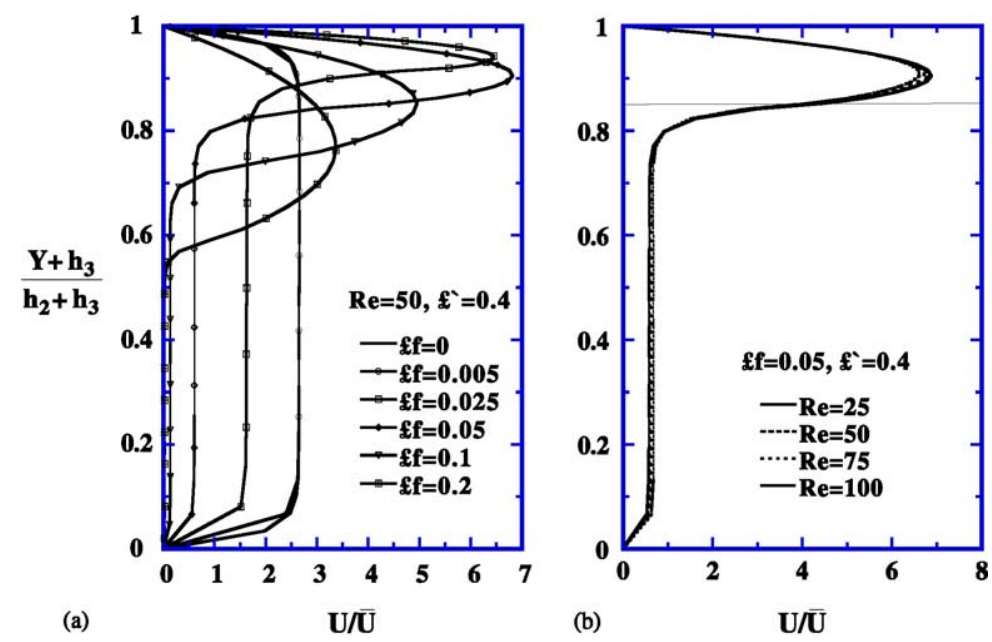

Fig. 7. Axial velocity distributions in the gap region and porous GDL layer at the central section of the blocked gap region. (a) Effects of gap size at $R e=50$ and $\varepsilon=0.4$; (b) effects of Reynolds number at $\lambda=0.05$ and $\varepsilon=0.4$.

\subsection{Effects of gap size and Reynolds number}

It can be expected that the change in flow area of the fuel channel may have a considerable influence on the mass transport in the flow channel and the GDL as well. The blocked channels with baffle of gap ratios $\lambda=0,0.005,0.05,0.1$ and 1 are investigated in the present work. For the cases of $R e=50$ and $\varepsilon=0.4$, Fig. 7(a) shows the blockage effects on the velocity profiles at the midway location $X=L_{1}+L_{2} / 2$ of the gap region between the baffle and the GDL. An abrupt change of velocity occurs at the interface except in the case of fully blocked channel $(\lambda=0)$, in which all of the fluid is forced to enter and flow through the GDL. As the flow channel is partially blocked $(0<\lambda<1)$, however, part of the fluid is allowed to move downstream through the gap and the rest is forced into the GDL. With the increase in the gap height, the mass flow rate through the gap increase but the gap flow velocity may reduce with the enlarged flow area. Due to the counteraction of these two effects, the gap flow velocity variation with the gap ration $\lambda$ is not monotonic. The data shown in Fig. 7 demonstrates that the axial velocity at $\lambda=0.05$ has a maximum among the cases considered. While the Reynolds number has only minor influence on the axial velocity profile in the gap region as shown in Fig. 7(b).

The amount of reactant gas entering the porous GDL is one of the most interested and significant flow quantities. Fig. 8 presents the ratio of the mass flow rate in porous GDL to the total mass flow rate, $\dot{m}_{\text {porous }} / \dot{m}_{\text {total }}$, at various flow and geometric conditions. It is observed that, in general, the numerical predictions of $\dot{m}_{\text {porous }} / \dot{m}_{\text {total }}$ are of reasonable agreement with the analytic approximations. From the results, it is also found that this fraction of the mass flow rate in the GDL reduces as the gap ratio increases. For a fixed value of $\lambda$, the fraction of the reactant gas transport in the GDL increases with increasing $R e$ since a higher pressure gradient, and thus the higher driving force, can be built in the presence of the baffle plate. The Re effects diminish at very small gap ratio $(\lambda \rightarrow 0)$ and very large ones $(\lambda \rightarrow 1)$ as well. Among the cases studied, the $R e$ effect at $\lambda=0.2$ is most noticeable.

Fig. 9 discloses effects of gap ratio and Reynolds number on the axial distribution of the oxygen mass flux, $\dot{m}_{1}^{\prime \prime}$, on the catalyst surface. In Fig. 9(a), the oxygen mass flux $\dot{m}_{1}^{\prime \prime}$ is shown at $R e=50$ and $\varepsilon=0.4$. The distribution of $\dot{m}_{1}^{\prime \prime}$ is characterized by a descending trend along the channel length but has a peak value at the location beneath the baffle plate. Larger amount of reactant mass is forced towards the catalyst surface by the presence of the baffle plate, and the smaller the gap size, the higher the mass flux is. However, changes of $\dot{m}_{1}^{\prime \prime}$ appearing in the upstream and downstream regions of the baffle plate are moderate. The oxygen mass flux in the channel can be enhanced by increasing $R e$ which gives a strong mass convection in the channel main flow, as shown in Fig. 9(b).

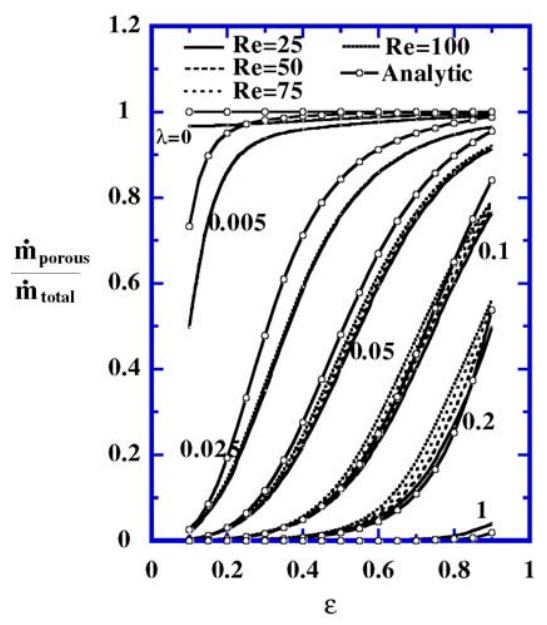

Fig. 8. Normalized mass flow rates in porous layer at the axial location of gap region center and at various conditions. 

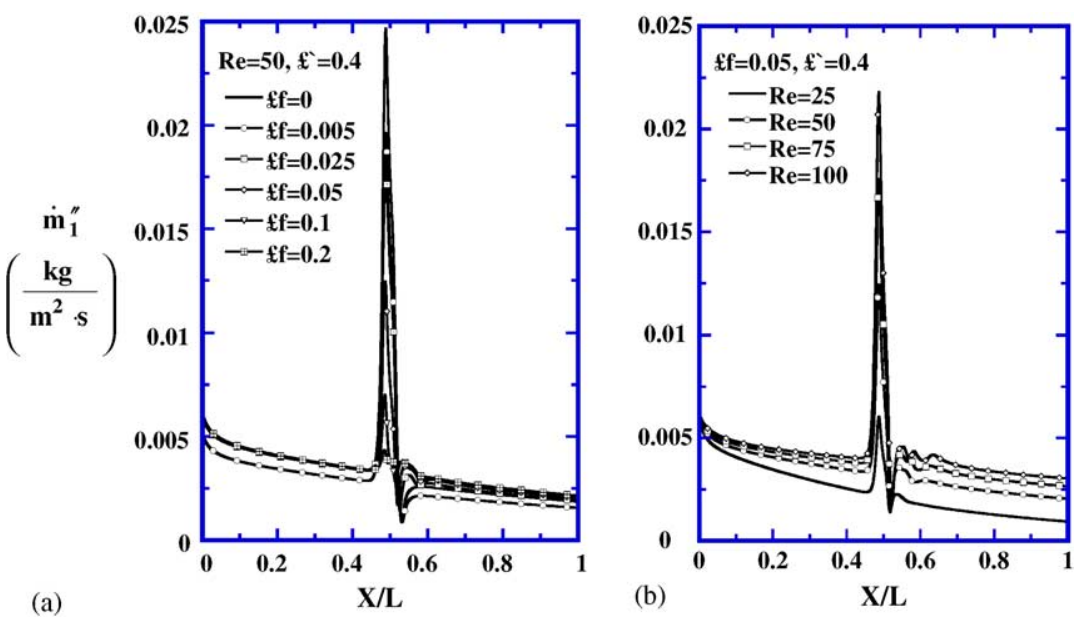

Fig. 9. Oxygen mass flux distributions at the surface of the catalyst layer. (a) Effects of gap size at $R e=50$ and $\varepsilon=0.4$; (b) effects of Reynolds number at $\lambda=0.05$ and $\varepsilon=0.4$.

\subsection{Effects of number of baffle plates on mass transport}

Number of the baffle plate is one of the significant parameters in the present analysis of transport phenomena. Fig. 10 shows the distributions of the oxygen flux $\dot{m}_{1}^{\prime \prime}$ along the interface between the catalyst layer and the GDL at $R e=50, \varepsilon=0.7$, and $\lambda=0,0.05$, and 0.1. Locally, the mass flux is of peak values at the locations corresponding to the baffle plates and the peak values decay downstream. With various numbers of the baffle plates, the cases with small gap ratio $\lambda$ generally have higher peak values of the oxygen mass flux under the first baffle. The total mass flow rate of the oxygen reaching the catalyst layer, $\dot{m}_{1}$, can be evaluated by the integration $\dot{m}_{1}=\int_{0}^{1} \dot{m}_{1}^{\prime \prime} \mathrm{d} x$, where $x=X / L$. Fig. 11 presents the values of $\dot{m}_{1}$ for the cases of $R e=50$ and $\varepsilon=0.7$ with various gap ratio $\lambda$ and baffle number $N$. Reduction in gap ratio causes an increase in $\dot{m}_{1}$ due to blockage effects of the baffle plates. Also, the data in Fig. 11 demonstrate that adding baffle plates enhances the oxygen mass transport toward the catalyst layer through GDL.

From the data shown in Fig. 10, it is obvious that the axial distributions of the oxygen mass flux $\dot{m}_{1}^{\prime \prime}$ at the interface of the GDL and the catalyst have a similar pattern. In Fig. 12, we plot the normalized mass flow rate $\dot{m}_{\text {porous }} / \dot{m}_{\text {total }}$ at the midway of the channel, $X / L=0.5$, under conditions of
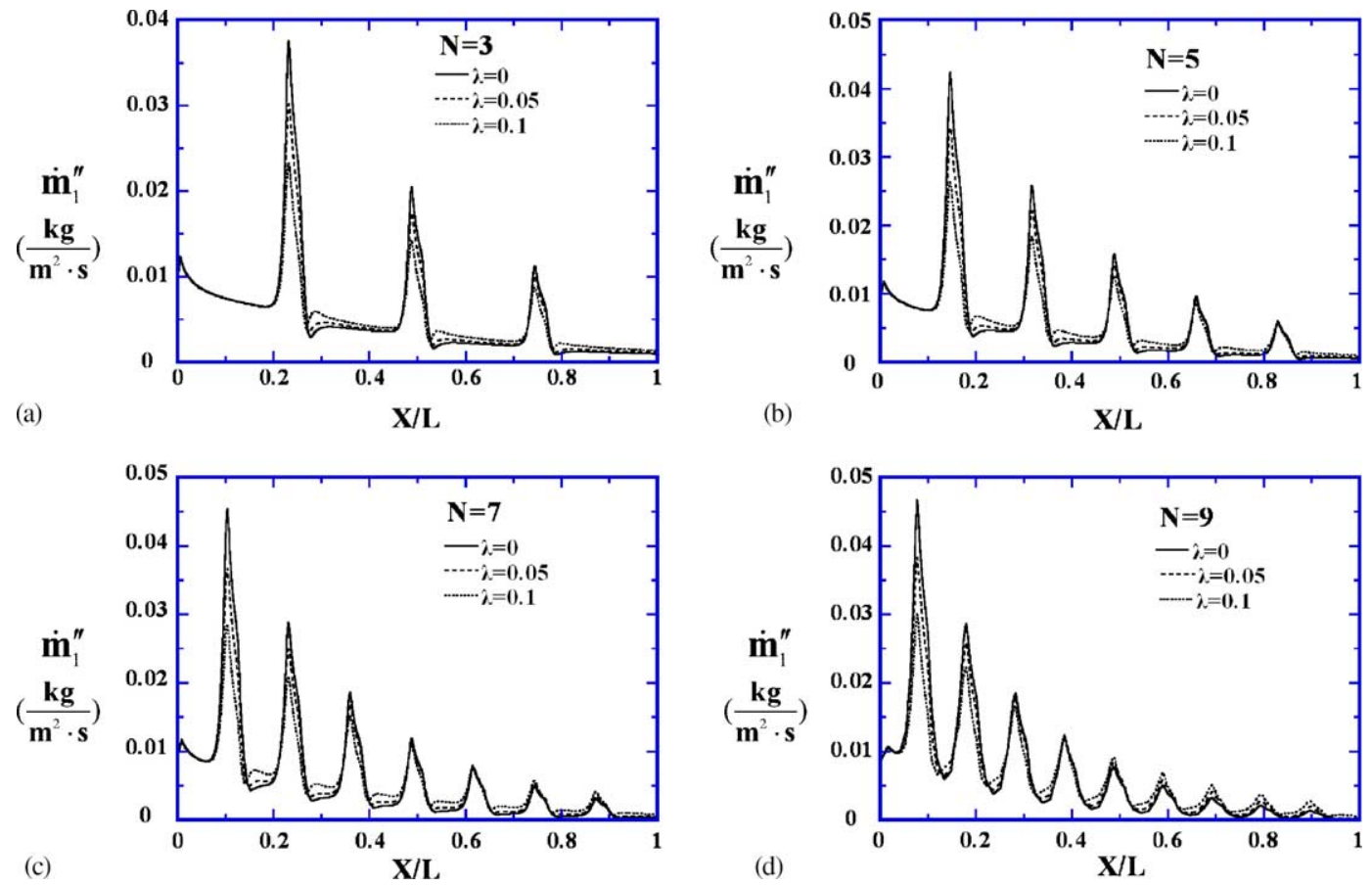

Fig. 10. Axial distributions of oxygen mass fluxes at $R e=50, \varepsilon=0.7$ and various values of gap size and baffle number. (a) $N=3$; (b) $N=5$; (c) $N=7$; (d) $N=9$. 


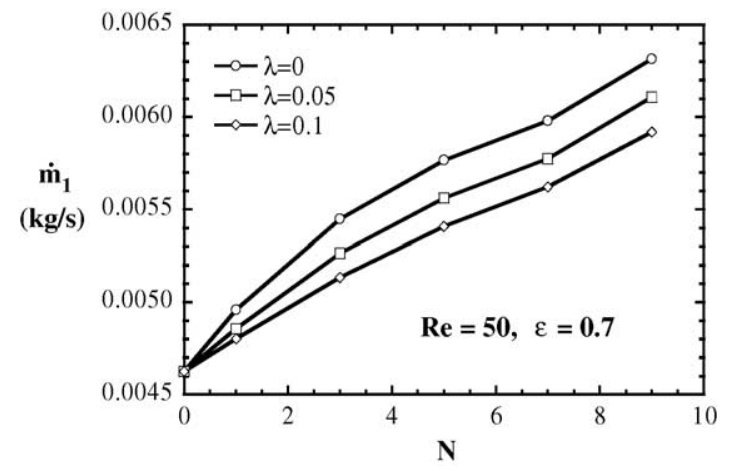

Fig. 11. Oxygen mass flow rate reaching the interface of GDL and catalyst layer for the cases of $R e=50, \varepsilon=0.7$ and various values of gap size and baffle number.

various combinations of porosity, gap ratio, and number of baffle plates to demonstrate the similarity. As the trend shown in Fig. 8 , at a larger gap ratio, the fraction $\dot{m}_{\text {porous }} / \dot{m}_{\text {total }}$ is lower since most of the fluid moves downstream through the gap between the baffle plates and the GDL, and a smaller amount of fluid is forced to pass through the porous GDL. On the other hand, the mass flux fraction increases with increasing porosity for the smaller flow resistance in the GDL. However, this special value of the mass flux fraction at the location $X / L=0.5$ is almost independent of the number of baffle plates.

To examine the influence of baffle plate number on the local distribution of water vapor concentration, contours at the surface of the catalyst layer for the cases of $R e=50, \varepsilon=0.4$, $\lambda=0.05$, and $N=3,5,7$, and 9 are shown in Fig. 13. Under the assumption of fast and complete reaction of the oxygen reaching the surface of the catalyst layer, the amount of the water generated directly depends on the local mass flux of the reactant gas, i.e., oxygen. It is observed that high water vapor concentration appears at the locations under the baffle plates where there is a high oxygen mass flux as shown in Fig. 10.

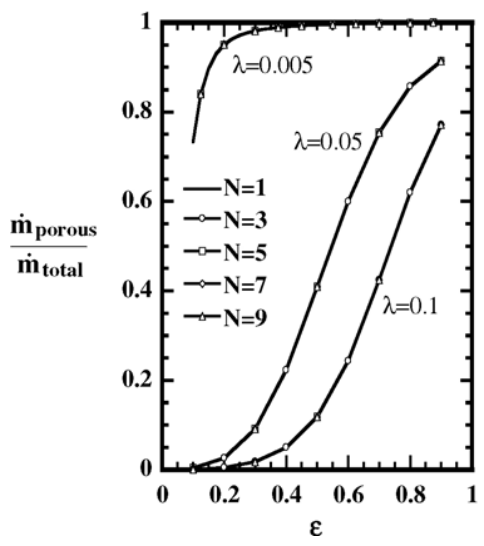

Fig. 12. Effects of baffle plate number, gap ratio $\lambda$ and porosity $\varepsilon$ on fraction of the mass flow rate entering the GDL at $X / L=0.5$.
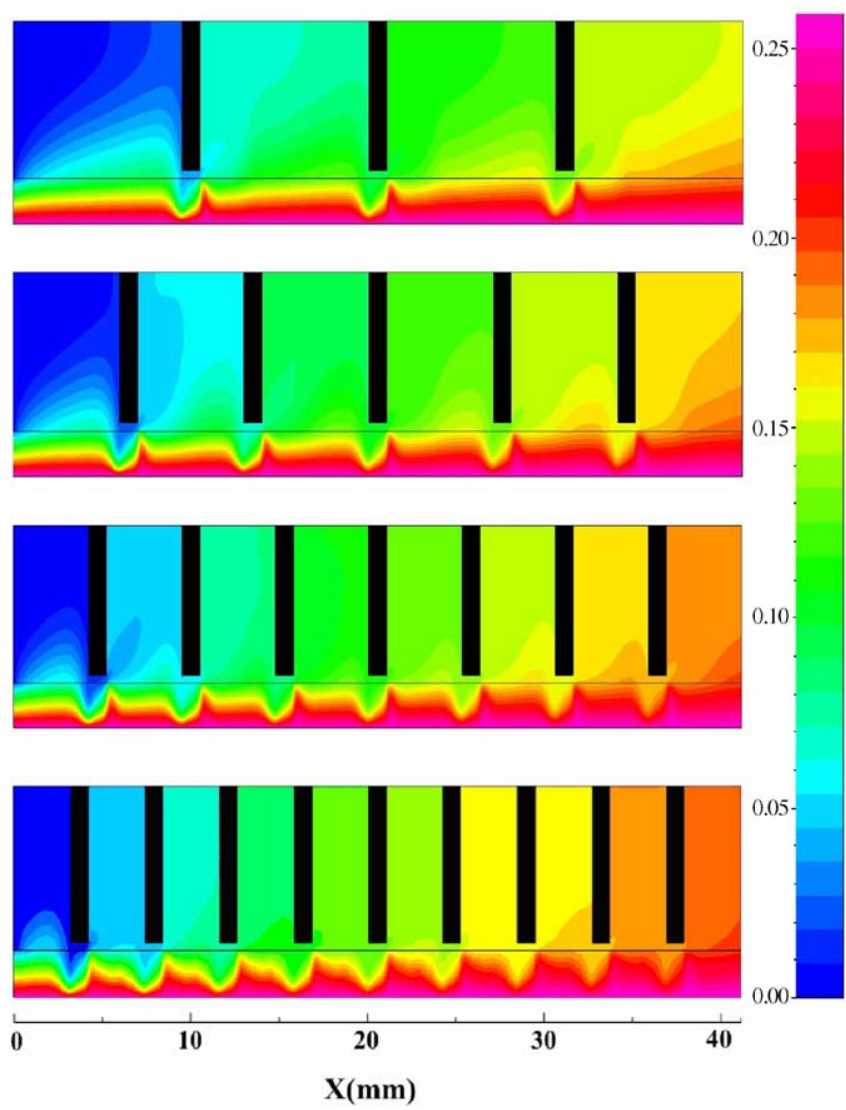

Fig. 13. Effects of baffle number on the local water vapor concentration in the baffle-gap-GDL region of the present composite flow passage at $R e=50$, $\lambda=0.05$ and $\varepsilon=0.4$.

\subsection{Pressure drop across the channel length}

The presence of the baffle plates placed normal to the main flow direction partially blocks the fuel channel and forces most of the fuel to go through the porous GDL and reach the catalyst interface. This arrangement improves the fuel transport rate and, therefore, the reaction at the catalyst layer can be enhanced. However, the intrusion of the baffle plates causes larger pressure-loss and needs higher pumping power for delivery of the fuel. Fig. 14 presents the influences of baffle gap ratio, main flow Reynolds number, and the number of baffle plates on the dimensionless pressure drop across the fuel channel, $\Delta P^{*} \equiv\left(P_{\text {inlet }}-P_{\text {exit }}\right) /\left(\rho U_{0}^{2} / 2\right)$. From the data in Fig. 14(a), it can be realized that the pressure drop increases with reducing gap size and/or porosity. With an appropriate gap between the baffle and the GDL, e.g., $\lambda=0.1$ or larger, the pressure drop and then the pumping cost needed would be considerably reduced. The data in Fig. 14(b) show that the dimensionless pressure drop reduces with the increasing main flow Reynolds number and the effects in the cases considered are moderate. This trend is similar to the theory of the laminar channel flow, in which the pressure drop is inversely proportional to the Reynolds number. Increasing the number of the baffle plates implies the stronger blockage effect and 


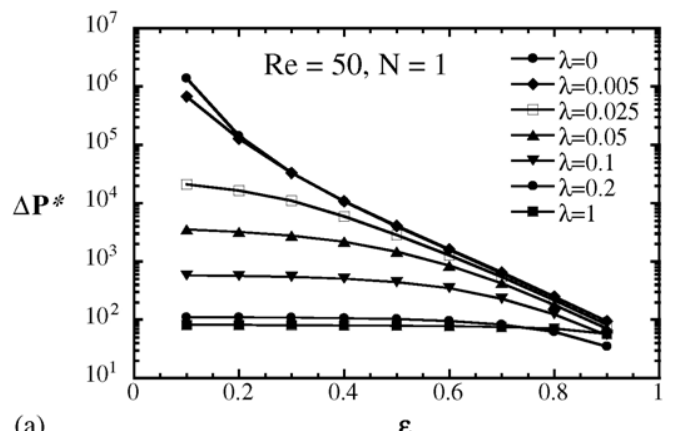

(a)

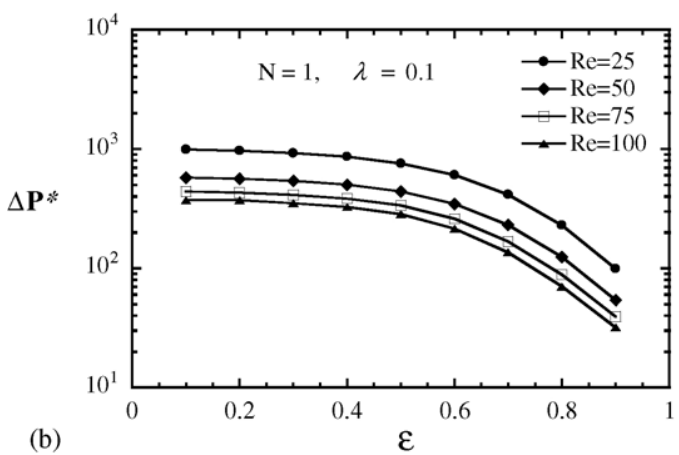

(b)

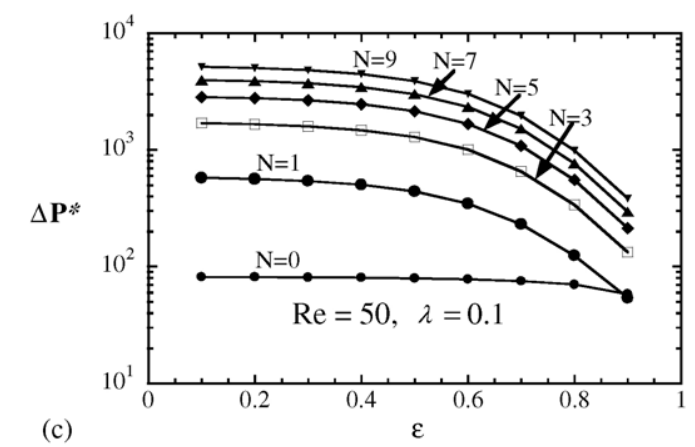

Fig. 14. Pressure drop in one-side porous-walled channel at various conditions. (a) Effects of baffle gap size for $R e=50$ and $N=1$; (b) effects of Reynolds number for $N=1$ and $\lambda=0.1$; and (c) effects of number of baffle plates for $R e=50$ and $\lambda=0.1$.

higher pressure-loss, as shown in Fig. 14(c). From the present results, it is found that extremely high pressure loss can be generated due to high flow resistance at a low gap ratio combined with a low GDL porosity. In general, the pressure drop in the fuel channel can be reduced noticeably with increasing porosity of GDL in the range of $\varepsilon>0.6$.

\section{Concluding remarks}

In the present study, we have investigated the reactant gas transport in a PEM fuel cell with the fuel channel partially blocked by installing baffle plates. The effects of the baffle blockage and the porosity of the GDL on the fuel gas flow and mass transfer have been examined in detail. Based on the present results and analysis, the following conclusions can be drawn.
The presence of the baffle plates has significant impact on the flow field in the fuel channel and the gas reactant transport through the GDL. The baffles block the main flow in the channel and force more fuel gas to turn to the GDL. This fact implies an enhancement of the oxygen mass flux and the water vapor concentration at the catalyst surface, especially at the positions right beneath the locations of the baffle plates. The simulation demonstrated that the diffusion layer of $\varepsilon=0.7$ is beneficial to the performance of the reactant gas transport. Increasing the number of baffles in the flow channel, the oxygen flux in the catalyst layer under the baffle would become higher. However, reducing gap size and/or increasing baffle number to enhance the reactant gas transport results in a penalty of high pressure-loss. Although the present model without three-dimensional relieving effect may exaggerate the pressure-loss, the qualitative trend of the present predictions is useful for evaluation of the penalty stemmed from high resistance due to the baffle blockage and the low GDL porosity. In the parameter ranges considered in the present work, the gap ratio has the most remarkable impact on the variation of the pressure drop. Extremely high pressure loss can be generated due to high flow resistance at a low gap ratio combined with a low GDL porosity and should be avoided. With the consideration of both high performance and reasonable pressure drop, the present results disclose that designs with the baffle gap ratio no smaller than 0.1 , number of baffle plates $N=3-5$, and the GDL porosity around 0.7 seem quite appropriate.

\section{Acknowledgements}

The present study was supported by the Industrial Technology Research Institute, Energy Commission, and the National Science Council, Taiwan, the Republic of China through the grants NSC-92-2212-E-035-027, NSC-92-2212-E-211-001, NSC 92-2212-E-002-096.

\section{References}

[1] A.C. West, T.F. Fuller, Influence of rib spacing in proton-exchange membrane electrode assemblies, J. Appl. Electrochem. 6 (1996) 557.

[2] L.R. Jordan, A.K. Shukla, T. Behrsing, N.R. Avery, B.C. Muddle, M. Forsyth, Diffusion layer parameter influencing optimal fuel cells performance, J. Power Sources 86 (2000) 250.

[3] L.R. Jordan, A.K. Shukla, T. Behrsing, N.R. Avery, B.C. Muddle, M. Forsyth, Effect of diffusion-layer on the performance of the polymer electrolyte fuel cells operating at atmospheric pressure, J. Appl. Electrochem. 30 (2000) 641

[4] V. Gurau, F. Barbir, H. Liu, An analytical solution of a half-cell model for PEM fuel cells, J. Electrochemchemical Soc. 47 (2000) 2468.

[5] E. Hontanon, M.J. Escudero, C. Bautista, P.L. Garcia-Ybarra, L. Daza, Optimisation of flow-field in polymer electrolyte membrane fuel cells using computational fluid dynamics techniques, J. Power Sources 86 (2001) 363 . 
[6] S. Dutta, S. Shimpalee, J.W. Van Zee, Numerical prediction of massexchange between cathode and anode channels in a PEM fuel cell, Int. J. Heat Mass Transfer 44 (2001) 2029.

[7] R.J. Kee, P. Korada, K. Walters, M. Pavol, A generalized model of the flow distribution in channel networks of planar fuel cells, J. Power Sources 109 (2002) 148.

[8] A. Kumar, R.G. Reddy, Modeling of polymer electrolyte membrane fuel cell with metal foam in the flow-field of the bipolar/end plates, J. Power Sources 114 (2003) 54.

[9] W.M. Yan, C.Y. Soong, F. Chen, H.S. Chu, Effect of flow distributor geometry and diffusion layer porosity on reactant gas transport and performance of proton exchange membrane fuel cells, J. Power Sources 125 (2004) 27.

[10] S.J. Lee, A. Chang-Chien, S.W. Cha, R. O’Hayre, Y.I. Park, Y. Saito, F.B. Prinz, Design and fabrication of a micro fuel cell ar- ray with 'flip-flop' interconnection, J. Power Sources 112 (2002) 410.

[11] C.Y. Wang, P. Cheng, Multiphase flow and heat transfer in porous media, Adv. Heat Transfer 30 (1997) 93.

[12] C.Y. Wang, W.B. Gu, B.Y. Liaw, Micro-macroscopic coupled modeling of batteries and fuel cells Part 1. Model development, J. Electrochemical Soc. (1998).

[13] F.A.L. Dullien, Porous Media, Academic Press, New York, 1991.

[14] T. Berning, D.M. Lu, N. Djilali, Three-dimensional computational analysis of transport phenomena in a PEM fuel cell, J. Power Sources 106 (2002) 284.

[15] S.V. Patankar, Numerical Heat Transfer and Fluid Flow, Hemisphere, New York, 1980.

[16] D.A. Nield, A. Bejan, Convection in Porous Media, Springer-Verlag, New York, 1992. 